\title{
Minimum Time Transition Between Quantum States in Gravitational Field
}

\author{
Branko Novakovic \\ Faculty of Mechanical Engineering and Naval Architecture, University of Zagreb, Zagreb, Croatia
}

Email address:

branko.novakovic@fsb.hr

\section{To cite this article:}

Branko Novakovic. Minimum Time Transition Between Quantum States in Gravitational Field. American Journal of Modern Physics. Vol. 10, No. 2, 2021, pp. 30-35. doi: 10.11648/j.ajmp.20211002.12

Received: April 1, 2021; Accepted: April 15, 2021; Published: April 23, 2021

\begin{abstract}
Here it is started with the proportionality between Planck's and related gravitational parameters. Using the ratio between Planck mass and related minimal gravitational radius (half of Planck length) we obtain maximal radial density $(\mathrm{kg} / \mathrm{m}$ ) in gravitational field. On the other hand, minimal radial density one obtains using the ratio between Planck mass and related maximal radius in gravitational field. It is based on new Relativistic Alpha Field Theory (RAFT) that predicts the existence of minimal and maximal gravitational radius in a gravitational field. Thus, no singularity at the minimal gravitational radius and no infinity at the maximal gravitational radius. It is shown that the maximal radial density is constant and is valid for all amounts of masses. Also, minimal radial density is constant and is valid for all amounts of masses. Using Planck parameters, it is calculated the energy conservation constant $k=0.999934$. Since this constant is less from unity and grater from zero, the minimal gravitational radius cannot be zero (no singularity in a gravitational field) and maximal gravitational radius cannot be infinitive (no infinity in gravitational field). Here quantization of a gravitational field is based on the multiplication of the minimal gravitational length (twice of minimal radius) by parameter $n=1,2, \ldots$ The calculation of the minimum time transition between two quantum state for the proton gives $0.413466 \times 10^{-62}$ seconds. The minimal expansion time from minimal to maximal radius of proton is equal to $1.253992 \times 10^{-58} \mathrm{sec}$. This is in accordance with recently observation, revealing nano big bang: the first millisecond of crystal formation. The calculation of the minimum time transition between two quantum state for Universe is $13.948503 \times 10^{9}$ years. The minimal expansion time from minimal to maximal radius of Universe is equal to $422,151.136168 \times 10^{9}$ years. Previous calculation is based on the velocity equal to the speed of light. Since the real transition velocity is less than the speed of light, the real transition and expansion times are greater compare to the previous calculation. Following the previous results, one can understand why the quantum approach has only sense for the small mases i.e. particles.
\end{abstract}

Keywords: Quantum States, Minimum Time Transition, Gravitational Field, Energy Conservation Constant, Planks Parameters

\section{Introduction}

A theoretically verification that only a non-Gaussianity approach can create a quantum theory of gravity is presented in previous study [1]. Namely, this approach gives a sufficient signature that a gravitational field can be quantized. Further, the problem of the minimum time evolution between two quantum states is also presented and discussed [2]. The quantum transition, time dependent perturbation theory, Fermi-Golden rule and impurity scattering are presented in [3]. On the other hand, the time-dependent perturbation theory is explained in [4]. One particle transition, classical to quantum transition, probabilities of transition in two level state, sudden transition, quantum critical points, dynamical quantum phase transitions and quantum thermodynamics are illustrated in [5-17].

In some applications of quantum theory, like quantum computing, we want to know what is the shortest physically possible time for a quantum state to evolve to another one? This is closely linked to dynamical characterization derived from the time-energy uncertainty relations [2]. In order to develop the algorithm for solution of the mentioned problem in quantum dynamics, one should use the time-energy uncertainty relation and generalize it in the case of a time-dependent Hamiltonian operator [18]. In the modern 
quantum theory, the generic Hermitian operator is used.

For the quantitative measure of the evaluation of the quantum control system performance, one can use parameter $\eta$. This parameter is a ratio of the minimal (shortest) time $\left(\tau_{\min }\right)$ for transition of quantum states and the time by which this transition can be measured by control system ( $\left.\tau_{\mathrm{CQS}}\right)$, or control algorithm. One of the possibilities to solve this problem is to employ the time-energy uncertainty relation obtained by Pfeifer [19]. An analytical approach to digital quantum computing model is the Fahri - Gutmann system which is based on a variation of the quantum search algorithm. This is similar to the well - known Grover's algorithm [20]. This approach can be applied by following the presented steps procedures [21, 22].

Recently, it is presented the unification of quantum and gravitational theories in $2 \mathrm{~d}$ system [23]. In this unification the related matrix model is discussed by using the thermal partition function of Jackiw-Teitelboim (JT) gravity and asymptotically Euclidean AdS2 background. It is shown that the partition function of JT gravity is written as the expectation value of a macroscopic loop operator in the old matrix model of $2 \mathrm{~d}$ gravity. It is happened in the background where infinitely many couplings are turned on in a specific way. New discretization of gravitational field is presented in the previous study [27]. Further it is discussed the possibility that positive gravitational force could be source of dark energy [28]. The tension between Planck's and other observations is also analyzed [29]. The loss of dark matter since the birth of the universe is elaborated in detail [30]. Laser test of the potential of the diffraction pattern is employed in order to prove of Einstein's Theory of Relativity [33].

Here it is considered the minimum time evolution between two quantum states in gravitational field. This approach is based on new Relativistic Alpha Field Theory (RAFT) [24-26]. In this theory each mass can be in the state of the minimal gravitational radius, as well as, in in the state of the maximal gravitational radius. Thus, the minimal gravitational radius can be the initial point for quantization of gravitational field and the maximal gravitational radius can be the final point of quantization of gravitational field. It is shown that the quantum approach in gravitational field has only sense for the small mases i.e. particles. The minimal expansion time of proton from minimal to maximal radius (for the expansion velocity equal to the speed of light) is equal to $1.253992 \times 10^{-58}$ sec. On the other hand, the minimal expansion time of Universe from minimal to maximal radius (for the expansion velocity equal to the speed of light) is equal to $422,151.136168 \times 10^{9}$ years. Since the real expansion velocity is less than the speed of light, the minimal expansion time for proton and Universe is greater compare to the previous calculations.

\section{Gravitational Parameters as Functions of Planck's Parameters and Solution of Energy Conservation Constant}

New Relativistic Alpha Field Theory (RAFT) [24-26] extends GR to the extremally strong gravitational field, including Planck scale. In RAF theory Energy Momentum Tensor (EMT) of gravitational field is generated from the left side of field equations [32]. Further, in RAF theory minimal gravitational length, or minimal gravitational diameter, for Planck mass is equal to Planck length. This means that the minimal gravitational radius of Planck mass is equal to half of Planck length for spherically symmetric mass. Further, the ratio of Planck mass and Planck length is constant, $M_{p} / L_{p}=c^{2} / G$. In gravitational field, the ratio of mass $M$ and minimal gravitational length $L_{g}$ is also constant $M / L_{g}=c^{2} / G$, and is equal to the ratio of Planck's mass and length. Following this consideration, the gravitational length, time, energy and temperature can be presented as the function of the Planck length, time, energy and temperature, respectively [31]:

$$
\begin{gathered}
L_{g}=L_{p} \frac{M}{M_{p}}=\sqrt{\hbar G / c^{3}} \frac{M}{M_{p}}, t_{g}=t_{p} \frac{M}{M_{p}}=\sqrt{\hbar G / c^{5}} \frac{M}{M_{p}}, \\
E_{g}=E_{p} \frac{M}{M_{p}}=\sqrt{\frac{\hbar c^{5}}{G}} \frac{M}{M_{p}}, T_{g}=T_{p} \frac{M}{M_{p}}=\sqrt{\frac{\hbar c^{5}}{G k}} \frac{M}{M_{p}} .
\end{gathered}
$$

Here $L_{g}, M, G, t_{g}, E_{g}$, and $T_{g}$ are gravitational length, mass, constant, time, energy and temperature, while $L_{p}, M_{p}, \hbar, t_{p}, E_{p}$ and $T_{p}$ are Planck's length, mass, constant, time, energy and temperature, respectively. The amounts of the Planck's parameters are given in the relations:

$$
\begin{aligned}
& L_{p}=1.616255 \cdot 10^{-35} \mathrm{~m}, M_{p}=2.176435 \cdot 10^{-8} \mathrm{~kg}, \\
& \hbar=1.054571 \cdot 10^{-34} \mathrm{~m}^{2} \mathrm{~kg} \mathrm{~s}^{-1}, t_{p}=5.391245 \cdot 10^{-44} \mathrm{~s} \text {, } \\
& E_{p}=1,956 \cdot 10^{9} J, T_{p}=1.416808 \cdot 10^{32} \mathrm{~K} .
\end{aligned}
$$

Following (1) we can introduce factor of proportionality $\left(K_{p}\right)$ equal to $K_{p}=M / M_{p}$. Appling (1) to proton in gravitational field, we obtain the related proton parameters:

$$
\begin{gathered}
L_{g p r}=1.242116 \cdot 10^{-54} \mathrm{~m}, M_{p r}=1.672622 \cdot 10^{-27} \mathrm{~kg}, \\
G=6.67408 \cdot 10^{-11} \mathrm{~m}^{3} \mathrm{~kg}^{-1} \mathrm{~s}^{-2}, t_{g p r}=4.143253 \cdot 10^{-63} \mathrm{~s}, \\
E_{g p r}=1.503215 \cdot 10^{-10} \mathrm{~J}, T_{g p r}=1.088838 \cdot 10^{13} \mathrm{~K} .
\end{gathered}
$$

Generally, following the relations (1), (2) and (3), one can conclude that the gravitational parameters (at the minimal radius) for the masses less than Planck mass are smaller than the related Planck's parameters. On the contrary, the gravitational parameters for the masses greater than Planck mass are larger than the related Planck's parameters.

In order to calculate the energy conservation constant $\kappa$, one can use Planck mass and Planck length:

$$
L_{\text {min }}=L_{p}=\frac{2 G M_{p}}{(1+\kappa) c^{2}}, \quad \kappa=\frac{2 G M_{p}}{L_{p} c^{2}}-1,
$$

$$
\kappa=0.99993392118 \text {. }
$$


The all spherically symmetric particles (bodies) in a gravitational field, with mass $M$, have maximal and minimal radial density at the minimal and maximal gravitational radiuses, respectively [21]:

$$
\begin{aligned}
& \rho_{\text {rmax }}=\frac{M}{r_{\text {min }}}=\frac{(1+\kappa) c^{2}}{G}=2.693182 \cdot 10^{27} \mathrm{~kg} / \mathrm{m}, \\
& \rho_{\text {rmin }}=\frac{M}{r_{\text {max }}}=\frac{(1-\kappa) c^{2}}{G}=0.888779 \cdot 10^{23} \mathrm{~kg} / \mathrm{m},
\end{aligned}
$$

Here $r_{\min }$ and $r_{\max }$ are minimal and maximal radiuses of mass $M, c$ is a speed of light in vacuum, $G$ is gravitational constant, and $\kappa$ is energy conservation constant from (4) (see [27]). Further, $\rho_{r \max }$ and $\rho_{r \min }$ are maximal and minimal radial density in a gravitational field valid for all amounts of masses.

Gravitational quantum effect for masses less than the Plank's mass is dominant in the region between $L_{\text {min }}$ and $2 L_{\text {min. }}$. Therefore, quantization of gravitational field shood be determined in the following region:

$$
\begin{gathered}
r_{\text {min }}=\frac{G M}{(1+\kappa) c^{2}}, 0<\kappa<1, L_{\text {min }}=2 r_{\text {min }}=\frac{2 G M}{(1+\kappa) c^{2}}, \\
2 L_{\text {min }}=\frac{4 G M}{(1+\kappa) c^{2}}, 2 L_{\text {min }}-L_{\text {min }}=\frac{2 G M}{(1+\kappa) c^{2}}, \\
\frac{2 L_{\text {min }}-L_{\text {min }}}{L_{d}}=n_{\text {max }}, \quad n=1,2, \ldots, n_{\text {max }} .
\end{gathered}
$$

Here $L_{d}$ is the minimal distance between two quantum points.

\section{Minimum Transition Time Between Two Quantum States in Gravitational Field}

Let $\Delta T$ is the shortest time during which the average value of a certain physical quantity is changed by an amount equal to the standard deviation. Thus $\Delta T$ can be called standard deviation, or uncertainty of time. This time should satisfy the following relation:

$$
\begin{gathered}
\Delta H^{\wedge} \Delta T \geq \frac{\hbar}{2}, \\
\Delta_{\psi} H^{\wedge}=\left(\left\langle\psi\left|H^{\wedge}\right| \psi\right\rangle-\left\langle\psi\left|H^{\wedge}\right| \psi\right\rangle^{2}\right)^{1 / 2} .
\end{gathered}
$$

Here $\Delta \hat{H}$ is the energy uncertainty. In the relation (7) quantum dynamical evolution is starting from a generic state $|\psi\rangle$ and is finishing in the related orthogonal state. The quantitative measure of temporal quantum state transfer efficiency $\eta_{t}$ is given by:

$$
\begin{gathered}
\eta_{t} \equiv \frac{t_{\text {min }}}{\tau_{C Q S}}, \quad \eta_{\psi \rightarrow \psi \perp} \equiv \frac{\tau_{\psi \rightarrow \psi \perp}}{\tau_{C Q S}}, \\
\eta_{\psi \rightarrow \psi \perp}=\frac{\pi \hbar}{2 \Delta H^{\wedge} \tau_{C Q S}}, \tau_{\psi \rightarrow \psi \perp} \geq \pi \hbar / 2 \Delta H^{\wedge} .
\end{gathered}
$$

Here $t_{\text {min }}$ is the shortest physically possible time to obtain the quantum transition between two quantum states. Parameter $\tau_{C Q S}$ can be stated as the time, effectively spent by the controlled system or control algorithm. Parameter $\tau_{\psi \rightarrow \psi \perp}$ is the shortest physically possible time that is spent for the transition to the orthogonal state $\psi_{\perp}$. This is the minimum transition time between two quantum points. For determination of the minimal time in quantum dynamical evolution, one should use time depended Hamiltonian. In that case, the time-energy uncertainty relation, obtained by Pfeifer [19], can be used.

For application of the previous theory to the quantum system one can start with the minimal distance between two quantum states $L_{d}$ given by (6). Let the transition velocity between two quantum points is denoted by $v$. In that case distance between two quantum points, $L_{d}$, can be determined by the relation:

$$
L_{d}=v \tau_{\psi \rightarrow \psi \perp}
$$

The most minimal distance between two quantum points, one obtains if the transition velocity between two quantum points is equal to the speed of light. In that case the most minimal distance between two quantum points can be calculated by the relation:

$$
v=c \rightarrow L_{d \min }=c \tau_{\psi \rightarrow \psi \perp} .
$$

Following the previous relations one can calculated the maximal number of quantum points in the region $2 L_{\min }-L_{\min }$ :

$$
n_{\max } \leq \frac{2 L_{\min }-L_{\min }}{c \tau_{\psi \rightarrow \psi \perp}} .
$$

From (6) and (11) one obtains the maximal number of quantum points in the region $2 L_{\min }-L_{\min }$ as function of the gravitational mass:

$$
n_{\max } \leq \frac{2 G M /(1+\kappa) c^{2}}{c \pi \hbar / 2 \Delta H^{\wedge}} .
$$

Thus, in gravitational field the maximal number of quantum points in the region $2 L_{\min }-L_{\min }$ are given by the relation:

$$
n_{\text {max }} \leq \frac{4 G M}{\pi \hbar c^{3}} \frac{\Delta H^{\wedge}}{(1+\kappa)} .
$$

As it can be seen from (13), the maximal number of quantum points is proportional to the gravitational mass. 
Further, including only equality relations in (7) and (8), one can obtain the following equation:

$$
\tau_{\psi \rightarrow \psi \perp} * n_{\max }=\frac{2 G M}{(1+\kappa) c^{3}} .
$$

Applying proton mass (3) to the relation (14) we obtain the numerical relation valid for proton:

$$
\tau_{\psi \rightarrow \psi \perp} * n_{\max }=\frac{0.826905 * 10^{-62}}{(1+\kappa)} \mathrm{sec} .
$$

\section{Comparison of Minimum Time in Quantum States Transitions Between Proton and Universe}

Now, including Universe mass into the relation (14) one obtains the following relation:

$$
\begin{gathered}
M=1.775786 * 10^{53} \mathrm{~kg}, \\
\tau_{\psi \rightarrow \psi \perp} * n_{\max }=\frac{0.8797309 * 10^{18}}{(1+\kappa)} \mathrm{sec} . \\
n_{\max }=\frac{0.8797309 * 10^{18}}{\tau_{\psi \rightarrow \psi \perp} *(1+\kappa)} \mathrm{sec} .
\end{gathered}
$$

Farther, taking parameter $\kappa$ from (4) and including into (16) we obtain the maximal number of quantum points in one minimal gravitational length $L_{\min }$ of the Universe:

$$
n_{\max }=\frac{0.439879 * 10^{18}}{\tau_{\psi \rightarrow \psi \perp}} \mathrm{sec} .
$$

Using $n=1$ and including parameter $\kappa$ from (4) one obtains the minimal transition time of Universe (16) from the minimal radius to the radius $r_{c} \cong 2 r_{\text {min }}$ :

$$
\text { Universe : } \tau_{\psi \rightarrow \psi \perp}=13.948503 \cdot 10^{9} \text { years. }
$$

Thus, the present age of Universe is close to the age that belongs to the radius $\left(r_{c} \cong 2 r_{\min }\right)$. On the other hand, the present Universe acceleration is close to the point $\left(\mathrm{r}_{\mathrm{c}} \cong 2 \mathrm{r}_{\mathrm{min}}\right)$ where it will change to the deceleration.

Farther, using parameter $\kappa$ from (4) and $n=1$ we obtain, from (14), the minimal transition time for proton from minimal radius to the radius $\left(\mathrm{r}_{\mathrm{c}} \cong 2 \mathrm{r}_{\text {min }}\right)$ :

$$
\text { Proton : } \tau_{\psi \rightarrow \psi \perp}=0.413466 \cdot 10^{-62} \mathrm{sec} \text {. }
$$

Comparing (18) and (19) one can understand why the quantum approach has only sense for the small mases i.e. particles.

Now, one can ask how many quantum transition times are there between minimal and maximal radius of a mass? The velocity equation for each mass expansion has two zeros. The first zero is at the minimal radius and second one is at the maximal radius [24-26]:

$$
r_{\min }=\frac{G M}{(1+\kappa) c^{2}}, \quad r_{\max }=\frac{G M}{(1-\kappa) c^{2}} .
$$

Following (14) and (20), the maximal number of quantum transition times between minimal and maximal gravitational radiuses can be calculated by the relation:

$$
n_{\max }=\frac{2 G M}{(1-\kappa) c^{3} \cdot\left(\tau_{\psi \rightarrow \psi \perp}\right)} .
$$

Including mass of the proton, parameter $\kappa$ from (4) and $\left(\tau_{\psi \rightarrow \psi \perp}\right)$ from (19), one obtains the following result:

$$
\left(n_{\max }\right)_{\text {proton }}=30,328.788
$$

On the other hand, including mass of Universe, parameter $\kappa$ from (4) and $\left(\tau_{\psi \rightarrow \psi \perp}\right)$ from (18), one obtains the maximal number of transition times between minimal and maximal radiuses of Universe:

$$
\left(n_{\max }\right)_{\text {universe }}=30,264.991
$$

From the previous analysis one can conclude that the ratio of the maximal transition times for the Universe and proton is constant and is equal to unity. Numerically it is close to unity:

$$
\begin{gathered}
\left(n_{\text {max }}\right)_{\text {univ. }}=\frac{2 G M_{\text {univ }}}{(1-\kappa) c^{3} \cdot\left(\tau_{\psi \rightarrow \psi \perp}\right)_{\text {un.first }}}, \\
\left(n_{\text {max }}\right)_{\text {prot. }}=\frac{2 G M_{\text {prot. }}}{(1-\kappa) c^{3} \cdot\left(\tau_{\psi \rightarrow \psi \perp}\right)_{\text {pr.first }}}, \\
\frac{\left(n_{\text {max }}\right)_{\text {univ. }}}{\left(n_{\text {max }}\right)_{\text {prot. }}}=0.997896 \approx 1 .
\end{gathered}
$$

Thus, the maximal number of the quantum transition times from the minimal to the maximal radiuses of the related mass is the same for all quantity of masses. But, the quantity of the one quantum transition time for the proton is some parts of the seconds, while for the Universe it is close to 14 billion years.

Now, using (1) we can calculate maximal length of the Universe at the maximal gravitational radius:

$$
\begin{gathered}
L_{\text {max }}=n_{\text {max }} L_{\text {min }}=n_{\text {max }} \sqrt{\hbar G / c^{3}} \frac{M}{M_{p}}, \\
L_{\text {max }}=3.0264991 \cdot 10^{4} * 1.3187268 \cdot 10^{26}, \\
L_{\text {max }}=3.991255 \cdot 10^{30} \mathrm{~m} .
\end{gathered}
$$

The minimal expansion time of Universe from minimal to the maximal radius (for the expansion velocity equal to the speed of light) is: 


$$
t_{\text {min.exp.univ }}=n_{\text {max }} \tau_{\psi \rightarrow \psi \perp}=422,151.136168 \cdot 10^{9} \text { years. }
$$

Since the expansion velocity is less than the speed of light, the expansion time of Universe is greater of $t_{\text {min.exp }}$ in (26).

The minimal expansion time of proton from minimal to the maximal radius (for the expansion velocity equal to the speed of light) is:

$$
t_{\text {min.exp.prot. }}=n_{\max } \tau_{\psi \rightarrow \psi \perp}=1.253992 \cdot 10^{-58} \mathrm{sec} .
$$

This is extremally small expansion time of proton that can be neglected in quantum calculation. Further, this is in accordance with recently observation [34] where the scientists were revealing nano big bang with the first millisecond of crystal formation.

Now, we are interested about maximal radial velocity in a gravitational field. In that sense, one can start from the relations of radial velocity and acceleration in gravitational field [29]:

$$
\begin{gathered}
\dot{r}= \pm\left[\frac{2 G M}{r}\left(1-\frac{G M}{2 r c^{2}}\right)+\left(\kappa^{2}-1\right) c^{2}\right]^{1 / 2}, \\
\ddot{r}=-\frac{G M}{r^{2}}\left(1-\frac{G M}{r c^{2}}\right) .
\end{gathered}
$$

As we know, the maximal radial expansion velocity in gravitational field is at point where the radial acceleration is equal to zero. Including this condition into (28) and taking $\kappa$ from (4), one obtains the following result:

$$
\begin{gathered}
\ddot{r}=0 \rightarrow\left(\frac{d r}{d t}\right)_{\max }=\dot{r}_{\max }= \pm \kappa c, \\
\dot{r}_{\max }= \pm 0.99993392118 \cdot c, \\
\dot{r}_{\max }= \pm 2.99772648 \cdot 10^{8} \mathrm{~m} / \mathrm{s} .
\end{gathered}
$$

Previous condition is occurred at the point where the gravitational radius is close to twice of the minimal radius $\left(\mathrm{r}_{\mathrm{c}} \cong 2 \mathrm{r}_{\text {min }}\right)$.

\section{Conclusion}

Here quantization of a gravitational field is based on the multiplication of the minimal gravitational length (twice of minimal radius). The calculation of the minimum time transition between two quantum state for the proton gives $0.413466 \times 10^{-62}$ seconds. On the other hand, the minimal expansion time from minimal to maximal radius of proton is equal to $1.253992 \times 10^{-58} \mathrm{sec}$. This is in accordance with recently observation, revealing nano big bang: the first millisecond of crystal formation. For the comparison, the calculation of the minimum time transition between two quantum state for Universe is $13.948503 \times 10^{9}$ years. The minimal expansion time from minimal to maximal radius of Universe is equal to $422,151.136168 \times 10^{9}$ years. Previous calculation is based on the velocity equal to the speed of light.
But the real transition velocity is less than the speed of light. Therefore, the real transition and expansion times are greater compare to the previous calculation. Following the previous results, one can understand why the quantum approach has only sense for the small mases i.e. particles. This article is based on new Relativistic Alpha Field Theory (RAFT). This theory predicts that there exist minimal and maximal gravitational radius in a gravitational field. Thus, no singularity at the minimal gravitational radius and no infinity at the maximal gravitational radius. This is consequence of existence of the energy conservation constant that here is calculated for obtaining a numerical amount.

\section{Acknowledgements}

This work was supported by the National Scientific Foundation of Republic of Croatia (grant number: 120-1201842-3048).

\section{References}

[1] R. Howl, V. Vedral, D. Naik, M. Christodoulou, C. Rovelli, and A. Iyer, Non-Gaussianity as a Signature of a Quantum Theory of Gravity, PRX Quantun 2, 010325 (2021).

[2] Lisboa A. C.; Piqueira J. R. C. Minimum Time in Quantum State Transitions: Dynamical Foundations and Applications, book: Research Advances in Quantum in Quantum Dynamics, Chapter 6, Publisher: InTech, Aug. (2016). DOI: $10.5772 / 63025$.

[3] Nanohub organization resources 5018. Time dependent perturbation. https://nanohub.org/resources/5018/

[4] Bantikum S. M. Transition probability of particle's Quantum State, Global Scientific Journal, GSJ: Vol. 6, Iss.-8, Aug. (2018). ISSN 2320-9186. www.globalscientificjournal.com

[5] Ruffo A. R. One Particle Transitions and Correlation in Quantum Mechanics. Journal of Research of the National Bureau of Standards-A. Physics and Chemistry Vol. 69A, No. 2, March-April 1965.

[6] Ruediger S. et al. A classical to quantum transition via key experiments. Eur. J. Phys. 41055304 (2020).

[7] Predominici-Scottle G., Palma J. Combining quantum mechanics and molecular mechanics. Same recent progresses in $\mathrm{QM} / \mathrm{MM}$ methods. Advances in Quantum Chemistry, (2010).

[8] Ohlsson T., Zhou, S. Transition probabilities in two level quantum System with PT symmetric nion Hermitian Hamiltonians. Journal of Mathematical Physics 61, 052104 (2020). https://doi.org/10.1063/5.0002958

[9] Lopez F. A. C., Allende S., Retamal S. Sudden transition between classical to quantum decoherence in bipartite correlated qutrit system. Scientific Reports, Vol. 7, 44654 (2017).

[10] Chen C., Chang A. M., Melloch M. Transition between quantum states in a parallel coupled double quantum dot. Physical Review Letters 17, 176801, DOI: 10.1103/PhysRevLett.92.176801. 
[11] Kreuz M. at all. A method to measure the resonance transitions between the gravitationally bound quantum states of neutrons in the GRANIT spectrometer. Nucl. Instr. Meth. A 611 (2009) 326 DOI: 10.1016/j.nima.2009.07.059.

[12] Remmy $\mathrm{Z}$ at all. Finding quantum critical points with neural network quantum states. 24th European Conference on Artificial Intelligence - ECAI 2020 Santiago de Compostela, Spain (2020).

[13] Transitions Between Energy States https://courses.physics.ucsd.edu/2017/Spring/physics4e/transit ions.pdf

[14] Zviagin A. A. Dynamical quantum phase transition. Low Temperature Physics 42, $971 \quad$ (2016). https://doi.org/10.1063/1.4969869

[15] Md. Manirul A., Huang Wei-Ming, Zhang Wei-Min, Quantum Thermodynamics of single Particle systems. Scientific Reports, vol. 10, 13500, (2020).

[16] Namkung M., Kwon Y. Almost minimum discrimination of $\mathrm{N}$-ary weak coherent states by Jaynes - Cummings Hamiltonian Dynamics. Scientific Reports, vol. 9, 19664 (2019).

[17] Kai Xu at. all. Probing dynamical phase transitions with a superconducting quantum simulator. Science Advances 17, vol. 6, no. 25, eaba4935 (2017). DOI: 10.1126/sciady.aba4935.

[18] Alessandro D. Introduction to Quantum Control and Dynamics CRC Press - Taylor \& Francis Group, Boca Raton, FLA, 2007.

[19] Pfeifer D. "How Fast Can a Quantum State Change with Time?" Physical Review Letters. v. 70, n. 22, 3365-3368, 1993.

[20] Grover L. K. Quantum Mechanics helps in searching for a needle in haystack. Phys. Rev. Lett. 79, 2, 325-328, (1997).

[21] Luo S. L. How fast can a quantum state evolve into a target state? Physica D: Nonlinear Phenomena. 189, 1, 1-7, 2004.

[22] Sakurai J. J. Modern Quantum Mechanics. Revised Edition. Addison Wesley Publishing, Reading, Mass. Company, Inc., 1994.

[23] K. Okuyamaa K.; Sakai K. JT gravity, KdV equations and macroscopic loop operators. SISSA, Springer, Jan. 24 (2020). https://doi.org/10.1007/JHEP01(2020)156

[24] Novakovic B. Relativistic Alpha Field Theory - Part I: Determination of Field Parameters. Inter. Jour. of New Technology and Research (IJNTR) ISSN: 2454-4116, Vol. 1, Iss. 5, Sept. (2015) https://doi.org./10.31871/IJNTR.15.15
[25] Novakovic B. Relativistic Alpha Field Theory - Part II: Does a Gravitational Field Could be Without Singularity? Inter. Jour. of New Technology and Research (IJNTR) ISSN: 2454-4116, $\begin{array}{llll}\text { Vol.-1, } & \text { Iss.-5, } & \text { Sept. } & \text { (2015) }\end{array}$ https://doi.org./10.31871/IJNTR.1.5.16

[26] Novakovic B. Relativistic Alpha Field Theory - Part III: Does Gravitational Force Becomes Positive if $(\mathrm{GM} / \mathrm{rc} 2)>1$ ? Inter Jour. of New Technology and Research (IJNTR) ISSN: 2454-4116, Vol.-1, Iss.-5, Sept. (2015) 39 https://doi.org./IJNTR.1.5.17

[27] Novakovic B. New Discretization of Gravitational Field. Inter. Jour. of New Technology and Research (IJNTR) ISSN: 2454-4116, Vol.-6, Iss.-12, Dec. (2020) 7. https://doi.org/10.31871/IJNTR.6.12.7

[28] Novakovic B. Is Positive Gravitational Force Source of Dark Energy? Inter. Jour. of New Technology and Research (IJNTR) ISSN: 2454-4116, Vol.-1, Iss.-7, Nov. (2015) 7. https://doi.org/10.31871/IJNTR.1.7.5

[29] Novakovic B. Could tension between Planck's and other observations be solved? Inter. Inter. Jour. of New Technology and Research (IJNTR) ISSN: 2454-4116, Vol.-6, Iss.-12, Dec. (2020) 7. https://doi.org/10.31871/IJNTR.6.9.18

[30] Rajna George. Fundamental Laws of Physics: Physicists measure the loss of dark matter since the birth of the universe. https://phys.org//hews/2016-12-physicists-loss-dark-birth-univ erse.html

[31] Novakovic B. Connection between Planck's and Gravitational Parameters. Inter. Jour. of New Technology and Research (IJNTR) ISSN: 2454-4116, Vol.-6, Iss.-12, Dec. (2020) 7. https://doi.org/10.31871/IJNTR.5.11.24

[32] Novakovic B. Energy Momentum Tensor Generates Repulsive Gravitational Force. Inter. Jour. of New Technology and Research (IJNTR) ISSN: 2454-4116, Vol.-5, Iss.-11, Nov. (2019) 45. https://doi.org/1031871/IJNTR.5.11.25

[33] Rajna George. Laser Proved Test of Einstein's Theory: The potential of the diffraction pattern. http://www.cartage.org.lb/en/themes/sciences/physics/Nuclear Physics/WhatisNuclear/Forces/Forces.htm

[34] Revealing nano big bang: Scientists observe the first millisecond of crystal formation. Lawrence Berkeley National Laboratory, Mar. (2021). https://phys.org/news/2021-03-revealing-nano-big-scientistsmilliseconds.html 